\title{
Dysregulated transcriptional networks in KMT2A- and MLLT10-rearranged T-ALL
}

\author{
Huining Kang ${ }^{1}$, Nitesh D. Sharma², Christian K. Nickl2, Meenakshi Devidas ${ }^{3}$, Mignon L. Loh ${ }^{4}$, Stephen P. Hunger ${ }^{5}$, \\ Kimberly P. Dunsmore ${ }^{6}$, Stuart S. Winter ${ }^{7}$ and Ksenia Matlawska-Wasowska ${ }^{2^{*}}$ (i)
}

\begin{abstract}
For children and young adults with T-lineage acute lymphoblastic leukemia (T-ALL), event free survival following relapse is $<10 \%$. We recently showed that rearrangements of the mixed lineage leukemia gene (KMT2A-R) are associated with induction failure and an inferior survival in T-ALL. Because there are currently no molecular features that inform treatment strategies in T-ALL, we hypothesized that transcriptional alterations related to KMT2A-R and MLLT1O-R T-ALL could identify biologically relevant genes and signaling pathways for the development of targeted therapies for these groups of patients. We analyzed microarray data from a retrospective cohort of $100 \mathrm{~T}$-ALL patients to identify novel targets for KMT2A $(n=12)$ or MLLT1O $(n=9)$ chimeras. We identified 330 probe sets that could discriminate between these groups, including novel targets, like RUNX2, TCF4 or MYO6. The results were further validated in two independent data sets and the functional networks were analyzed to identify pathways that may be of pathogenic or therapeutic relevance.
\end{abstract}

Keywords: Gene expression, KMT2A, MLLT10, Leukemia, Microarray, T-ALL

\section{Introduction}

Despite our growing understanding of the heterogeneity of genomic alterations in T-ALL [1-5], genetic alterations are not used to stratify therapy in T-ALL. Although, approximately $80-90 \%$ of patients with T-ALL can be cured, for those who relapse, event free survival is poor [6]. Because attempts to further dose-intensify therapy have generally resulted in greater toxicity without improved survival, efforts are underway to identify more effective treatments for patients with high-risk disease, including those with high levels of post-induction minimal residual disease [6]. A better understanding of the molecular drivers of resistant disease may inform the development of targeted therapies to improve outcome and reduce the burden of treatment-related acute and chronic adverse events.

The repertoire of chromosomal rearrangements affecting the KMT2A gene (KMT2A-R) includes over 100 translocation partners [7]. We recently showed that KMT2A-MLLT4 and del3'KMT2A are important determinants of high-risk disease in $H O X A$-deregulated T-ALL

\footnotetext{
* Correspondence: kmatlawska-wasowska@salud.unm.edu

${ }^{2}$ Department of Pediatrics, University of New Mexico, MSC105590,

Albuquerque, NM 87131, USA

Full list of author information is available at the end of the article
}

[8]. The clinical outcome of other HOXA-related lesions involving MLLT10 gene (MLLT10-R), remains controversial [8-10]. Using gene expression profiles, others have developed signatures that distinguish KMT2A-R in AML and B-ALL $[11,12]$ however such studies have not been performed on larger data sets for T-ALL. The data on transcriptional signatures in T-ALL with $K M T 2 A-\mathrm{R}$ and $M L L T 10-\mathrm{R}$ is very limited [2-5]. Specifically, gene expression signatures in $K M T 2 A-\mathrm{R}$ T-ALL were reported for only three KMT2A-MLLT1 cases thus far $[2,5]$. While deregulation of $H O X A 9 / 10$ is a hallmark of $K M T 2 A-R$ and $M L L T 10-R$, its over-expression does not inform patient outcome, suggesting that additional genes deregulated by these translocations could play an important role in leukemia pathobiology. Therefore, we hypothesized that supervised profiling of 100 well-characterized T-ALL cases could identify KMT2A- and MLLT10-deregulated genes and signaling networks allowing the development of targeted therapies in T-ALL.

\section{Material and methods}

Primary T-ALL samples were obtained from patients enrolled in Children's Oncology Group AALL0434 study $(n=100)$ [8]. All cases that passed the hybridization 
quality controls were subjected to Affymetrix U133 Plus 2.0 microarray [13]. Scale factor < 40; GAPDH M33197 $3^{\prime}$ ' intensity > 15,000; and GAPDH M33197 3'/5' ratio < 3 were applied as array experimental quality parameters [13]. The Robust Multi-array Average (RMA) algorithm was used to generate and normalize signal intensities $[8,13]$. From 54,675 probe sets, we selectively filtered out probes associated with gender-related genes, globins, and internal controls $[8,13]$. Linear Models for the Microarray approach implemented in $\mathrm{R}$ package limma [14] was utilized to identify differentially expressed probe sets in association to specific genomic lesions. Benjamini and Hochberg method was used to calculate the False Discovery Rate (FDR) to adjust for multiple testing. Java Treeview [15] was used to generate the heatmaps. Gene Set Enrichment Analysis (GSEA) [16] was performed to identify signaling pathways related to specific genomic lesions.

\section{Results and discussion}

Because KMT2A-R and MLLT10-R drive HOXA-deregulated leukemias, we sought to identify specific genes that are enriched in T-ALL with these genomic abnormalities. Specifically, we searched for differentially expressed genes that could discriminate between T-ALL cases with KMT2A-R $(n=12)$, MLLT10-R $(n=9)$ and the remaining T-ALL cases lacking these alterations (Others; $n=79$ ), and found 330 probe sets corresponding to genes deregulated between these groups (False Discovery Rate; FDR $\leq 0.05$ ) (Additional file 1: Figure S1A, Additional file 2: Table S1). For T-ALL samples harboring KMT2A-R, 258 probes sets were found significantly differentially expressed including 242 probe sets that were upregulated and only 16 probe sets corresponding to genes downregulated in KMT2A-R (Fig. 1a). In addition to $H O X A$ genes, $K M T 2 A-\mathrm{R}$ had increased expression of $P R O M 1$, encoding transmembrane glycoprotein, MYO6, which encodes ATP-dependent motor protein, and multiple regulators of transcription: RUNX2, TCF4, ZNF503, ZNF827, SMAD1, CPEB2. We also identified increased expression of WHAMMP2/WHAMMP3 and GOLGA8I pseudogenes located on chromosome 15q13.1, which were recently reported in AML [7]. Thirty-nine probe sets were upregulated and only one was downregulated in MLLT10-R cases (Fig. 1b). In agreement with others, MLLT10-R differentially expressed HOXA genes, MEIS1, and other genes located at chromosome 10: CASC10, SKIDA1, SPAG6, ZNF503, BMI1 and COMMD3 [4]. $Q K I$, which encodes an RNA-binding protein involved in alternative splicing, was found to be the most downregulated gene in both KMT2A-R and MLLT10-R cases (Fig. 1a, b). All the above genes except for BMI1, COMMD3, NKX2.3 and EML were also deregulated in $K M T 2 A-\mathrm{R}$ cases indicating that KMT2A-R and
MLLT10-R share similar transcription programs when compared to lesion-negative cases.

Because KMT2A-R and MLLT10-R demonstrate a strong similarity in $H O X A$-mediated deregulation of gene expression, we compared gene expression profiles between these two groups. Among 38 probe sets shown in Fig. 1c, thirty-two were downregulated and five were upregulated in MLLT10-R compared to KMT2A-R. MYO6, RUNX2, CPEB2, ZNF827 and TCF4 were the most overexpressed genes in $K M T 2 A-\mathrm{R}$ compared to MLLT10-R, suggesting that KMT2A-R T-ALL encompass a specific biological subset, which collectively drive a unique oncogenic program. Since KMT2A-MLLT4 confers an inferior outcome, we sought to determine which genes discriminate between KMT2A-MLLT4 $(n=5)$ and KMT2A-MLLT1 $(\mathrm{n}=5)$. Among the 26 discriminatory probes sets, we found two, MLLT4 and uncharacterized $R P 11-38 P 22$ that were over-expressed and 24 were downregulated, including SEPW1, SMAD1, CHI3L2 and MYOM2 between MLLT4 and MLLT1 (Additional file 1: Figure S1B).

To validate our findings in an independent patient cohort we have performed differential gene expression profiling using existing T-ALL microarray data sets reported by Soulier [2] and Dik [3]. The Soulie's data set consisted of 92 T-ALL cases including 3 harboring KMT2A-MLLT1 and four with PICALM-MLLT10 alterations. The Dik's data set comprised of 23 cases including six PICALM-MLLT10 fusions. We observed a significant overlap between our data and existing microarray data sets confirming that in T-ALL, KMT2A and MLLT10 chimeras drive unique transcriptional programs resulting in specific changes in gene signatures and expression patterns (Additional file 3: Table S2, Additional file 4: Table S3).

To further characterize transcriptional alterations in MLLT10-R and KMT2A-R T-ALL, we performed GSEA to assess functional networks and aberrant cell signaling pathways [16]. Gene ontology and canonical pathway analyses identified multiple genes and signaling networks, which were commonly or exclusively dysregulated in KMT2A-R and/or MLLT10-R cases. KMT2A-R were negatively enriched in regulators of protein export, intracellular protein localization and transport (Fig. 2a). Aberrant localization of oncoproteins or tumor suppressors have been detected in many different types of cancer [17]. Thus, downregulation in protein transport machinery may lead to the disruption in signal transduction in KMT2A-R T-ALL. On the contrary, genes upregulated in KMT2A-R included regulators of extracellular matrix organization and collagen formation, which are known modulators of cancer invasion (Additional file 5: Table S4). The tumor microenvironment and adhesion were also shown to play a protective role in conferring drug resistance in leukemia [18]. 


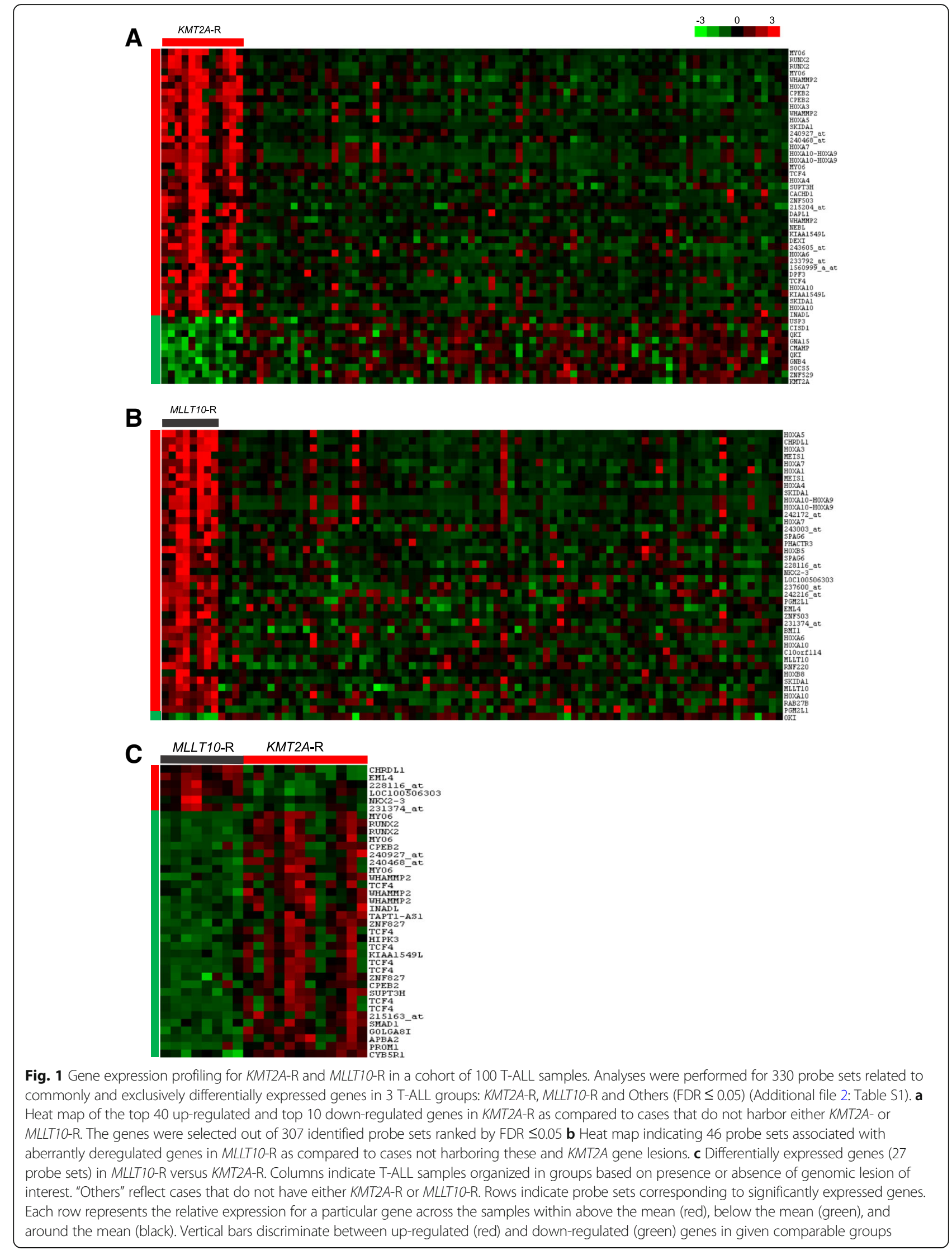



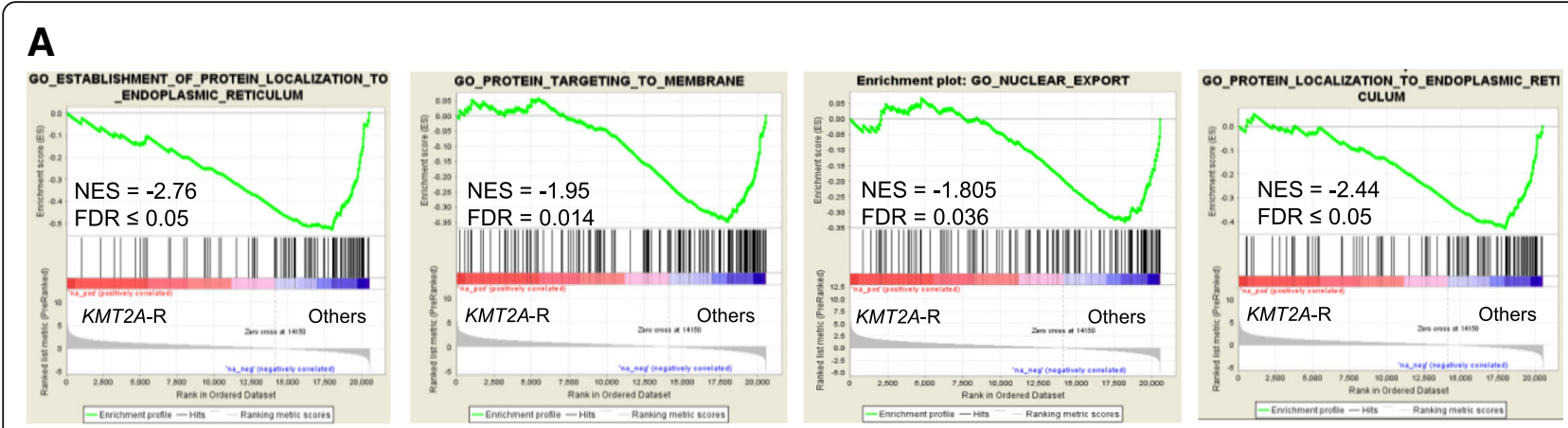

\section{B}
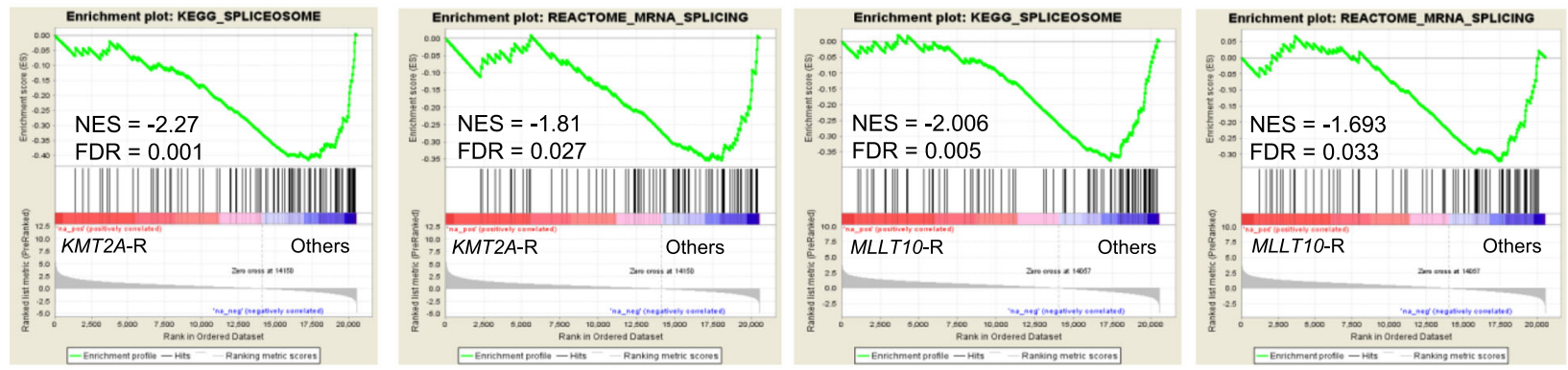

\section{C}
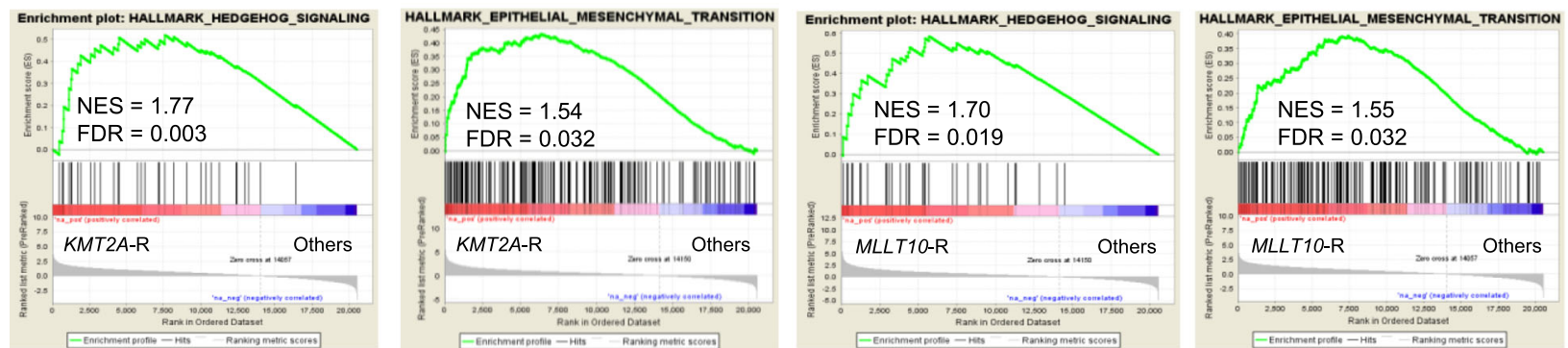

D
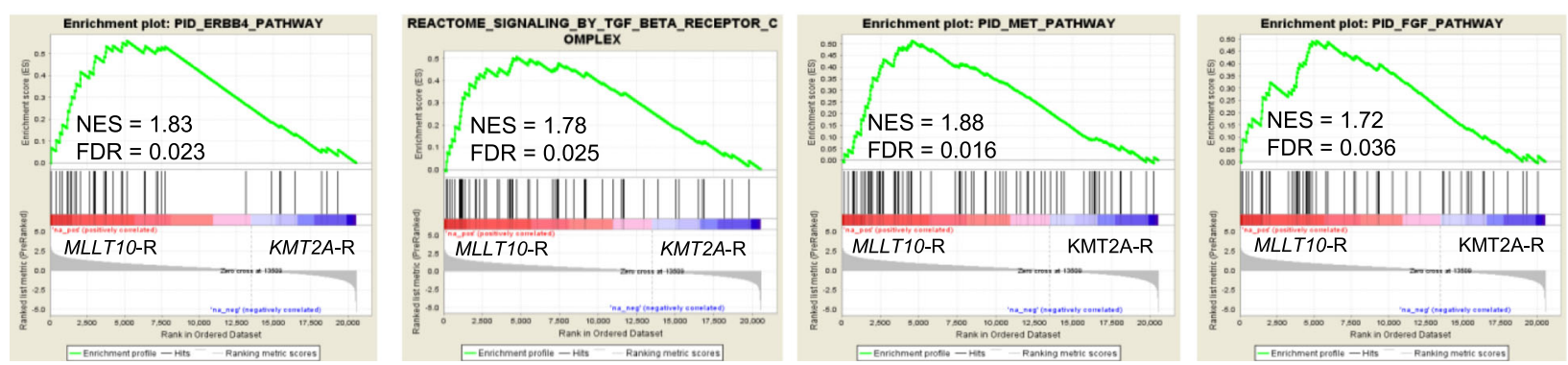

Fig. 2 KMT2A-R and MLLT1O-R T-ALL show dysregulation in multiple cell signaling pathways critical for leukemia development and progression. a Negatively correlated gene sets in KMT2A-R T-ALL (intracellular transport). Green line shows the enrichment score based on hits of genes (indicated by the bars on the abscissa) in the ordered list of differentially regulated genes resulting from the comparison of KMT2A-R positive samples and Other T-ALL patient samples. Red/blue bar area beneath the black bars indicates degree of association with a specific phenotype. b KMT2A-R and MLLT10-R are negatively enriched in genes involved in alternative splicing compared to the Others. c Example of positively enriched gene sets containing genes upregulated in both KMT2A-R and MLLT10-R samples compared to the Others. $\mathbf{d}$ Positively correlated gene sets in MLLT10-R vs. KMT2A-R. Gene sets containing genes upregulated in MLLT10-R positive samples compared to KMT2A-R samples (distinct tyrosine kinase signaling pathways). NES, Normalized Enrichement Score; FDR, False Discovery Rate 
Interestingly, MLLT10-R were positively enriched in regulators of embryonic development, while genes specifically downregulated in this group were mostly associated with cell cycle, DNA synthesis and repair (Additional file 5: Table S4, Additional file 6: Table S5) [4]. Genes involved in protein K48-linked ubiquitination and stem cell differentiation were positively enriched in MLLT10-R when compared to KMT2A-R (Additional file 7: Figure S2). Both, MLLT10-R and KMT2A-R showed downregulation in a number of genes involved in the regulation of gene expression, ribosome organization and biogenesis, and chromatin modifications (Additional file 5: Table S4, Additional file 6: Table S5). Importantly, we found that $K M T 2 A-\mathrm{R}$ and MLLT10-R were negatively enriched in genes involved in alternative splicing and mRNA processing (Fig. 2b). These findings might be linked to the downregulation of the $Q K I$ gene as seen in lung and brain tumors [19]. Deregulation of alternative splicing promotes genomic instability leading to the generation of aberrantly spliced genes and subsequent malignant transformation [20].

Recently, a subset of T-ALL cases with the activation in the hedgehog pathway was shown to be sensitive to the hedgehog pathway inhibitor, vismodegib [21]. The activation of hedgehog signaling has also been associated with increased cell proliferation and tumor resistance in several solid tumors [22]. Here, we show that KMT2A-R and MLLT10-R were positively enriched in genes encoding members of the hedgehog signaling network (Fig. 2c), indicating that patients harboring these lesions might benefit from therapies with hedgehog inhibitors. While the mechanisms mediating epithelial to mesenchymal transition (EMT) have been widely studied in solid tumors, several lines of evidence indicate a critical role of EMT modulators in promoting leukemia cell motility and migration [23]. Our data demonstrate positive enrichment in genes associated with EMT in KMT2A-R, suggesting the need to further investigate the roles of EMT in T-ALL progression and resistance (Fig. 2c).

While our findings demonstrated that KMT2A-R share common biological networks with MLLT10-R [4], KMT2A-R also shared similar gene expression signatures with $K M T 2 A-\mathrm{R}$ in AML or BCP-ALL [5, 11, 12]. Our GSEA results demonstrate a strong enrichment for published data sets of genes differentially expressed in KMT2A-R leukemias (Additional file 8: Figure S3). We also identified signaling networks that could discriminate between KMT2A-R and MLLT10-R T-ALL. Compared to KMT2A-R, MLLT10-R showed upregulation in multiple receptor and/or tyrosine kinase-mediated pathways (e.g. Met, TGFB, PYK2, ERBB, PDGFR), for which FDA-approved inhibitors are available (Fig. 2d, Additional file 6: Table S5). These findings are of great interest considering that MLLT10-R were associated with T-ALL relapse [10]. In summary, this study reports findings for 12 T-ALL cases with $K M T 2 A-\mathrm{R}$, which extends upon the observations by others $[2,5]$. Overall, we have identified an extended repertoire of aberrant gene expression profiles in $K M T 2 A-\mathrm{R}$ and MLLT10-R T-ALL. These findings provide a mechanistic basis for additional pre-clinical testing in classes of therapeutic agents that may hold promise for high-risk T-ALL.

\section{Additional files}

Additional file 1: Figure S1A. KMT2A-MLLT4 and KMT2A-MLLT1.
(PDF $169 \mathrm{~kb}$ )

Additional file 2: Table S1. Probe sets for KMT2A-R and MLLT10R. (PDF 372 kb)

Additional file 3: Table S2. Validation of gene expression for KMT2A-R. (PDF 446 kb)

Additional file 4: Table S3. Validation of gene expression for MLLT10-R. (PDF 243 kb)

Additional file 5: Table S4. GSEA for gene ontology. (PDF 265 kb)

Additional file 6: Table S5. GSEA for canonical pathways. (PDF 276 kb)

Additional file 7: Figure S2. Genes positively enriched in MLLT10.

(PDF $107 \mathrm{~kb}$ )

Additional file 8: Figure S3. KMT2A-R in B-ALL and AML. (PDF 231 kb)

\begin{abstract}
Acknowledgements
We would like to acknowledge the Analytical and Translational Genomics Shared Resources at UNM Comprehensive Cancer Center and the COG Cell Bank for the provision of patient samples.
\end{abstract}

\section{Funding}

This project was supported in part by the National Center for Research Resources and the National Center for Advancing Translational Sciences of the NIH through Grant number 8UL1TR000041, and by the National Cancer Institutes and the Cancer Center Support Grant through Grant Number P30CA118100. Other grant support: Dedicated Health Research Funds from the University Of New Mexico School Of Medicine (Matlawska), NIH U10 CA98543 (COG Chair's grant), Gabrielle's Angel Foundation (Kang), U10 CA98413 (COG Statistics and Data Center grant), and U24 CA114766 (Specimen Banking), U10 CA180886 (COG Operations Center), U10 CA180899 (COG Statistics and Data Center).

Availability of data and materials

Microarray data are deposed in GEO (GSE70536).

\section{Authors' contributions}

Conception and design; KMW. Administrative support and provision of study materials or patients; MLL, SPH, MD, KPD, RSL, SSW. Collection and assembly of data; HK, SSW, KMW. Data analysis and interpretation; HK, KMW, NDS, CKN. Manuscript writing and final approval of manuscript; all authors.

\section{Ethics approval and consent to participate}

All patients or their parent(s)/guardian(s) provided written, informed consent for future research in accordance with the Declaration of Helsinki and local institutional guidelines. The study was approved by the Human Research Review Committee at the University of New Mexico Health Sciences Center (IRB \#03-183).

\section{Consent for publication}

Not applicable.

\section{Competing interests}

Hunger: Merck: Equity Ownership; Sigma Tau: Consultancy; Jazz Pharmaceuticals: Consultancy; Spectrum Pharmaceuticals: Consultancy. The remaining authors have no conflicts to disclose. 


\section{Publisher's Note}

Springer Nature remains neutral with regard to jurisdictional claims in published maps and institutional affiliations.

\section{Author details}

'Department of Internal Medicine, University of New Mexico, Albuquerque, NM, USA. ${ }^{2}$ Department of Pediatrics, University of New Mexico, MSC105590, Albuquerque, NM 87131, USA. ${ }^{3}$ Department of Biostatistics, University of Florida, Gainesville, FL, USA. ${ }^{4}$ Department of Pediatrics, University of California at San Francisco, San Francisco, CA, USA. ${ }^{5}$ Children's Hospital of Philadelphia, University of Pennsylvania, Philadelphia, PA, USA. 'Pediatric Hematology/ Oncology, University of Virginia, Charlottesville, VA, USA. ${ }^{7}$ Children's Minnesota Research Institute, Children's Minnesota, Minneapolis, MN, USA.

Received: 21 May 2018 Accepted: 29 July 2018

Published online: 23 August 2018

\section{References}

1. Liu Y, et al. The genomic landscape of pediatric and young adult T-lineage acute lymphoblastic leukemia. Nat Genet. 2017;49(8):1211-8.

2. Soulier J, et al. HOXA genes are included in genetic and biologic networks defining human acute T-cell leukemia (T-ALL). Blood. 2005;106(1):274-86.

3. Dik WA, et al. CALM-AF10+ T-ALL expression profiles are characterized by overexpression of HOXA and BMI1 oncogenes. Leukemia. 2005; 19(11):1948-57.

4. Mulaw MA, et al. CALM/AF10-positive leukemias show upregulation of genes involved in chromatin assembly and DNA repair processes and of genes adjacent to the breakpoint at 10p12. Leukemia. 2012;26(5): 1012-9.

5. Ferrando $A A$, et al. Gene expression signatures in MLL-rearranged T-lineage and B-precursor acute leukemias: dominance of HOX dysregulation. Blood. 2003;102(1):262-8.

6. Asselin BL, et al. Effectiveness of high-dose methotrexate in T-cell lymphoblastic leukemia and advanced-stage lymphoblastic lymphoma: a randomized study by the Children's oncology group (POG 9404). Blood. 2011;118(4):874-83.

7. Lavallee VP, et al. The transcriptomic landscape and directed chemical interrogation of MLL-rearranged acute myeloid leukemias. Nat Genet. 2015; 47(9):1030-7.

8. Matlawska-Wasowska K, et al. MLL rearrangements impact outcome in HOXA-deregulated T-lineage acute lymphoblastic leukemia: a Children's oncology group study. Leukemia. 2016;30(9):1909-12.

9. Lo Nigro L, et al. Detection of PICALM-MLLT10 (CALM-AF10) and outcome in children with T-lineage acute lymphoblastic leukemia. Leukemia. 2013; 27(12):2419-21.

10. Ben Abdelali $R$, et al. The prognosis of CALM-AF10-positive adult T-cel acute lymphoblastic leukemias depends on the stage of maturation arrest. Haematologica. 2013;98(11):1711-7.

11. Ross ME, et al. Gene expression profiling of pediatric acute myelogenous leukemia. Blood. 2004;104(12):3679-87.

12. Mullighan CG, et al. Pediatric acute myeloid leukemia with NPM1 mutations is characterized by a gene expression profile with dysregulated HOX gene expression distinct from MLL-rearranged leukemias. Leukemia. 2007;21(9): 2000-9.

13. Kang $\mathrm{H}$, et al. Gene expression profiles predictive of outcome and age in infant acute lymphoblastic leukemia: a Children's oncology group study. Blood. 2012;119(8):1872-81.

14. Ritchie ME, et al. Limma powers differential expression analyses for RNAsequencing and microarray studies. Nucleic Acids Res. 2015:43(7):e47.

15. Saldanha AJ. Java Treeview--extensible visualization of microarray data. Bioinformatics. 2004;20(17):3246-8.

16. Subramanian A, et al. Gene set enrichment analysis: a knowledge-based approach for interpreting genome-wide expression profiles. Proc Natl Acad Sci U S A. 2005;102(43):15545-50.

17. Hill R, et al. Targeting nucleocytoplasmic transport in cancer therapy. Oncotarget. 2014;5(1):11-28.

18. Winter SS, et al. Enhanced T-lineage acute lymphoblastic leukaemia cell survival on bone marrow stroma requires involvement of LFA-1 and ICAM-1. Br J Haematol. 2001;115(4):862-71.

19. de Miguel FJ, et al. A large-scale analysis of alternative splicing reveals a key role of QKI in lung cancer. Mol Oncol. 2016;10(9):1437-49.
20. Adamia S, et al. A genome-wide aberrant RNA splicing in patients with acute myeloid leukemia identifies novel potential disease markers and therapeutic targets. Clin Cancer Res. 2014;20(5):1135-45.

21. Dagklis A, et al. Hedgehog pathway activation in T-cell acute lymphoblastic leukemia predicts response to SMO and GLI1 inhibitors. Blood. 2016;128(23):2642-54.

22. Hanna A, Shevde LA. Hedgehog signaling: modulation of cancer properies and tumor mircroenvironment. Mol Cancer. 2016;15:24.

23. Stavropoulou V, et al. MLL-AF9 expression in hematopoietic stem cells drives a highly invasive AML expressing EMT-related genes linked to poor outcome. Cancer Cell. 2016;30(1):43-58.
Ready to submit your research? Choose BMC and benefit from:

- fast, convenient online submission

- thorough peer review by experienced researchers in your field

- rapid publication on acceptance

- support for research data, including large and complex data types

- gold Open Access which fosters wider collaboration and increased citations

- maximum visibility for your research: over $100 \mathrm{M}$ website views per year

At $\mathrm{BMC}$, research is always in progress.

Learn more biomedcentral.com/submissions 